\title{
Übersetzung, Veredas: Lebensformen des übersetzten literarischen Werkes
}

\author{
Der Tod ist eine Blume, die blüht ein einzig Mal. \\ Doch so er blüht, blüht nichts als er. \\ Er blüht sobald er will, er blüht nicht in der Zeit. \\ Paul Celan ${ }^{1}$
}

Die Figur der Veredas (dt. Pfade), die Rosa in all seinen Werken heraufbeschworen und zusammengeführt hat, verweist auf die vielen Orte, die dieser Signifikant benennt, aber auch auf alles, was in den Räumen geschieht, denen dieser Name eine neue Bedeutung verleiht, ausgehend von dem, was verheißungsvoll vorhergesagt wird, bis hin zu dem, was sich als unheilvoll herausstellt. Folgt man wörtlich der Lehre der Buritis, den lebendigsten, verkörpern die roseanischen Veredas gleichzeitig die vielen Möglichkeiten, einen Weg einzuschlagen. Wenn also die Veredas den großen Sertão geografisch umreißen und seine Konturen und Räume gestalten, geben sie auch den Erfahrungen einer jeden Kreuzung eine Form, machen Wege möglich und erzeugen verschiedene Formen des Wanderns, ähnlich wie Heideggers Holzwege. ${ }^{2}$ Daher vielleicht die Kraft, die bereits im Titel Grande sertão: veredas verkündet wurde: eine Kraft, die nicht einfach poetisch im ästhetisch-literarischen Sinne, sondern auch poietisch ist, im Sinne all dessen, was sich als Sertão-Welt eröffnet, in jeder Wegabweichung, die sich eigens als Weg herausstellt und die in einem magischen Trick - einer Sorte Magie, die der Magie des Lebens angehört - Form annimmt und zum Kunstwerk wird: Veredas.

Von den Veredas der Übersetzung eines literarischen Werkes in diesem Sinne zu sprechen, bedeutet, von verschiedenen Arten des Lebens und Zusammenlebens zu sprechen, aber auch von einer Möglichkeit, Leben zu schenken und zu schaffen. Indem sie unter der Wirkung intensiven und umfangreichen Lesens auf einmalige Weise gelebt wird, auf die keine Übersetzungsgeste verzichten kann, beschreibt jede Übersetzung das Werk geographisch stets auf ihre eigene Weise.

1 Paul Celan: Die Gedichte aus dem Nachlass. In: Paul Celan: Die Gedichte. Kommentierte Gesamtausgabe, org. Barbara Wiedemann. Frankfurt: Suhrkamp 2003, S. 433-557.

2 Holz lautet ein alter Name für Wald. Im Holz sind Wege, die meist verwachsen jäh im Unbegangenen aufhören. Sie heißen Holzwege. Jeder verläuft gesondert, aber im selben Wald. Oft scheint es, als gleiche einer dem anderen. Doch es scheint nur so. Holzmacher und Waldhüter kennen die Wege. Sie wissen, was es heißt, auf einem Holzweg zu sein. Martin Heidegger: Holzwege. Frankfurt am Main: Vittorio Klostermann 1977.

כ Open Access. @ 2020 Mauricio Mendonça Cardozo, publiziert von De Gruyter. @) By-NC-ND Dieses Werk ist lizenziert unter der Creative Commons Attribution-NonCommercial-NoDerivatives 4.0 Lizenz. https://doi.org/10.1515/9783110677713-013 
Und wenn die Übersetzung während, wegen und für ihre Erarbeitung nicht von der Erfahrung (und der Arbeit) befreit werden kann, die Konturen und Räume des übersetzten Werkes in der anderen Sprache zu zeichnen und dabei ihre möglichen Wege zusammenzutragen und ihre eigenen Formen zu bilden, ist Übersetzen nicht nur eine einmalige Weise, um dem ursprünglichen Werk Leben einzuhauchen, sondern auch eine Art, eine einmalige Lebensform zu schaffen diese Denkfigur fasst die wichtigste Frage zusammen, die in diesem Essay entwickelt werden soll.

\section{Benjamin, das Leben des literarischen Werks und das Leben des übersetzten, literarischen Werks}

Im klassischen Essay Die Aufgabe des Übersetzers skizziert Walter Benjamin, wie einige seiner Kommentatoren hervorheben, bei der Entwicklung der Schwerpunkte seiner Überlegungen zur Übersetzung auch eine wichtige Reflexion über die Frage des Lebens des Kunstwerks. ${ }^{3}$ Dazu unternimmt er eine kurze Problematisierung des Lebensbegriffs und präzisiert dessen Bedeutung.

Der Zusammenhang zwischen Leben und Werk soll nicht als einfache Metapher verstanden werden: „In völlig unmetaphorischer Sachlichkeit ist der Gedanke vom Leben und Fortleben der Kunstwerke zu erfassen. “4 - eine Empfehlung, die uns noch deutlicher auffällt, wenn wir uns daran erinnern, wie intensiv und organisch der Autor die verschiedensten Metaphern verwendet, um sein übersetzerisches Denken in diesem Essay zu artikulieren. Das heißt, im Falle des Kunstwerkes, wenn es von der Figur des Lebens her gedacht wird, ist es für Benjamin nicht nur ein als ob, eine bildhafte oder allegorische Rede von dem Kunstwerk als Lebensform; im Gegenteil, der Autor wird auf der Idee bestehen, dass es sich tatsächlich um ein Leben handelt, ,in völlig unmetaphorischer Sachlichkeit‘, wie er selbst sagt.

Gleichzeitig verschiebt Benjamin mit diesem Schritt den Lebensbegriff von einer rein biologischen und natürlichen Dimension in eine historische. Für den Autor gilt:

3 Walter Benjamin: A tarefa do tradutor, übersetzt von Susana K. Lages. In: Escritos sobre mito e linguagem (1915-1921), Präsentation, Organisation und Anmerkungen von Jeanne Marie Gagnebin, übersetzt von Susana K. Lages und Ernani Chaves. São Paulo: Duas Cidades, Editora 34, 2011, p.101-119. Hier wird nach folgender deutscher Ausgabe zitiert Walter Benjamin: Die Aufgabe des Übersetzers. In: Iluminationen. Frankfurt am Main: Suhrkamp 1994, S. 50-62.

4 Benjamin: Die Aufgabe des Übersetzers, S. 51f. 
Vielmehr nur wenn allem demjenigen, wovon es Geschichte gibt und was nicht allein ihr Schauplatz ist, Leben zuerkannt wird, kommt dessen Begriff zu seinem Recht. Denn von der Geschichte, nicht von der Natur aus [...] ist zuletzt der Umkreis des Lebens zu bestimmen. ${ }^{5}$

Es ist wichtig, hier ebenso wie Jeanne Marie Gagnebin in Anmerkung 44 zur brasilianischen Übersetzung von Susana Kampff Lages darauf hinzuweisen, ${ }^{6}$ dass durch diese Verschiebung des Lebensbegriffs von einer einfachen natürlichen Einschreibung zu einer historischen Einschreibung Benjamin auch beginnt, zwischen einer Lebensform in ihrem bloßen natürlichen Ausdruck (das bloße Leben) und derjenigen zu unterscheiden, die dem Denker für seine Diskussion wichtiger zu sein scheint: eine Lebensweise, die ihren Zweck nur außerhalb von sich selbst findet, auf einer anderen Ebene, auf der Ebene der Geschichte.

Vor diesem Hintergrund der erkenntnistheoretischen Abgrenzung arbeitet Benjamin mit den bekannten Unterschieden zwischen Leben, Überleben und dem Fortleben des Kunstwerks. Die Übersetzung stammt daher in diesem Zusammenhang weniger aus dem Leben als aus dem Überleben des Originals - eine Unterscheidung, die angesichts der oben erwähnten Neudimensionierung des Lebensbegriffs noch mehr Konsistenz gewinnt. Mit anderen Worten, die Übersetzung würde sich weniger aus der bloßen wesentlichen Faktizität des ursprünglichen Werks (aus der bloßen Tatsache, dass es geschrieben und veröffentlicht wurde) ergeben als aus der Fähigkeit, die Grenzen dieser Faktizität historisch zu überschreiten und so ein Stadium seines Fortlebens zu bestimmen.

Was ich in dieser viel diskutierten Bewegung des benjaminischen Denkens hervorheben möchte, bezieht sich auf eben diese entscheidende Dimensionierung des Lebens, um die es bei dieser Art des übersetzerischen Denkens geht. Wenn Benjamin über das Kunstwerk spricht, bezieht er sich auf das Leben des Kunstwerks (oder des literarischen Werks, im engeren Sinne), insbesondere in seinem Ausdruck des Überlebens und Fortlebens. Wenn Benjamin jedoch beginnt, von der Übersetzung des literarischen Werks zu sprechen, dreht es sich noch immer um das Leben des Originalwerkes, es handelt sich ausschließlich um das Leben des Originalwerkes.

Mit anderen Worten, obwohl der Denker die Übersetzbarkeit zur wesentlichen Eigenschaft des literarischen Werkes erhebt und der Übersetzung einen eigenen Status als Form zuspricht, siedelt Benjamin diese Eigenschaft sowie ihre Kraft als Gesetz im Originalwerk an. In diesem berühmten Essay von Benjamin hat also das Leben der Übersetzung als solches keinen Platz, weder im Sinne seiner eigenen vi-

5 Ebd., S. 52.

6 Benjamin: A tarefa do tradutor, S. 106. 
talen Faktizität noch im Sinne einer dieser Faktizität eigenen Transzendenz im historischen Sinne; die Übersetzung ist allenfalls an den Grenzen eines simplen Ausdrucks des Lebens bemessen, aber eines Lebens, welches immerzu das Leben eines anderen ist.

In genau diesem Maße sagt der Autor im Übrigen, dass die Übersetzungen dem Original nichts bedeuten und dass sie im Gegenteil ihre Existenz dem Fortleben des Originals verdanken. Benjamin geht diesbezüglich sogar so weit, kategorisch zu werden: „In ihnen [den Übersetzungen] erreicht das Leben des Originals seine stets erneute späteste und umfassendste Entfaltung. “7 Mit anderen Worten: In Benjamins Reflexionen existiert die Übersetzung, sie erfüllt ihre Funktion, sie ist als Form konstituiert, aber weder als etwas, das zu einem eigenen Leben fähig ist, noch als etwas, das sich als lebenswürdige Form konstituiert ${ }^{8}$, die zur Transzendenz fähig wäre.

Abgesehen von jenen seltenen Ausnahmefällen (die berühmten Übersetzungen von Luther, Voss, Hölderlin usw.), bei denen sich Benjamin das Recht vorbehält, sie aus derselben Perspektive zu betrachten wie die Originalwerke - man beachte diese Besonderheit -, wird die Übersetzung in der benjaminschen Dimensionierung zu einer Art lebender Toter, die unversöhnlich der vampiresken Handlung des Originalwerkes ausgeliefert ist.

Wenn wir darin einen Aspekt von Benjamins Gedanken zur Übersetzung erkennen können, der unter dem Gesichtspunkt eines zeitgenössischen Blickes genauer überlegt werden sollte, ist jedoch $\mathrm{zu}$ beachten, dass es hier nicht darum geht, von Benjamin eine konzeptuelle Dimension der Übersetzung zu fordern, die uns zeitgenössischer wäre als ihm. Obwohl es ihm gelungen ist, Debatten voranzutreiben, die in ihrer eigenen Zeit weit über den Stand der Übersetzungsdiskussion hinausgegangen und die bis heute hochaktuell sind, kann man die Tatsache nicht ignorieren, dass es sich um ein Denken handelt, das auf gewisse Weise auch in der Zeit seiner Entstehung verankert ist (1921). Die innere Kohärenz und die starken Wirkungen dieses Denkens unter Berücksichtigung seines Einflusses handelt es sich doch um einen der vielleicht wichtigsten Essays, die jemals zur Übersetzung des literarischen Werkes geschrieben wurden - befreit den zeitgenössischen Kritiker nicht davon, den Dissonanzen zu begegnen, die sich in die-

7 Benjamin: Die Aufgabe des Übersetzers, S. 50.

8 Es sollte daran erinnert werden, dass unter den vielen metaphorischen Figuren Benjamins in diesem Aufsatz die vitalistischste, diejenige der Beziehung zwischen Schale und Frucht, eine Beziehung zur Sprache betrifft, die ausschließlich dem Bereich des Originals angehört; Im Falle der Übersetzung wird eben dieses Verhältnis zur Sprache von Benjamin durch die Figuren des königlichen Umhangs und des Königs allegorisiert - wenn der König ein lebender Körper ist, ist der Mantel, wie edel er auch ist, eine leblose Instanz. 
sem Gedanken zu erkennen geben, wenn dieser ausgehend vom aktuellen Stand der Forschung auf dem Gebiet der Theorie und Philosophie der Übersetzung gelesen wird.

Es muss auch daran erinnert werden, dass das Erkennen der Notwendigkeit der Auseinandersetzung mit dem trivialen Eindruck, dass der übersetzte Text die Lebenszeit des Originals beeinflusst - wie auch andere Formen derivativer, sprachlicher Produktion, etwa Kritik und Kommentar - nicht notwendigerweise eine Gleichgültigkeit gegenüber den Bedeutungen dieses Eindrucks impliziert. Wenn wir mit dem Flugzeug reisen, haben wir manchmal den Eindruck, dass wir uns sehr langsam bewegen, und das Wissen, dass wir mit einer immensen Geschwindigkeit unterwegs sind, beeinflusst auch diesen Eindruck kaum. Was wir heutzutage nicht tun können, ist, diese Frage zu ignorieren und weiterhin mit der Idee zu leben, dass eine Vorrangstellung der Beziehung zwischen Original und Übersetzung (auch wenn dies gerechtfertigt ist) zwangsläufig das (heute nicht mehr vertretbare) Verbot jeder anderen relationalen, zeitlichen und vitalen Dimensionierung des übersetzten Textes impliziert.

\section{Exkurs: Die Infantia der Übersetzung}

Für ein bestimmtes Verständnis der Übersetzung - ein Verständnis, das trotz aller reflexiven Bemühungen der letzten Jahrzehnte leider nicht nur Laien oder dem sogenannten common sense zugeschrieben werden kann - würde vom übersetzten Text nichts anderes erwartet als die Aufgabe zu vollbringen, das Originalwerk nachzuerzählen. Und da in den Idealbegriffen, in denen eine solche Ökonomie der Übersetzung artikuliert wird, normalerweise nichts jenseits der eindeutigen Dimensionierung dieses Nacherzählens akzeptiert wird, erscheint unter dieser Perspektive als einzig akzeptable Stimme der Übersetzung die Stimme des Originals, die Stimme des Anderen in der Übersetzung. Mit anderen Worten, der Übersetzung wird weder die Möglichkeit noch das Recht eingeräumt, eine eigene Stimme zu haben. Das nenne ich hier die Infantia der Übersetzung, im Sinne einer vermeintlichen Schwierigkeit oder Unfähigkeit zu sprechen (In-fantia) oder einfach in dem Sinne, ein Recht nicht zu erkennen, für sich selbst zu sprechen, eine eigene Stimme zu haben, wie dies zum Beispiel der Fall bei Kindern ist. Auf Portugiesisch kann man junge, noch nicht volljährige Menschen als «infantes» bezeichnen.

Wenn wir jedoch nur minimal konsequent sind mit der komplexeren Dimensionierung, die die Konzepte von Subjekt und Sprache in der Aktualität erreichen, woraus die Anerkennung des Übersetzens als kritischer und transformierender Aktivität und des übersetzten Werks als singulärem Objekt erfolgt - der im Übrigen allein unter diesem Singularitätszustand in der Lage ist, seine Bestimmung 
als Übersetzung eines anderen Textes zu erfüllen -, sind wir verpflichtet, die Notwendigkeit eines Bruchs mit diesem Zustand der Infantia anzuerkennen, die das Übersetzen bis hierhin in einer idealisierenden und idealisierten Auffassung dieser Praxis gefangen hielt.

Wenn es einerseits keinen Grund gibt, die Idee, dass die Übersetzung das ursprüngliche Werk wiedergibt, vollkommen aufzugeben, führt der Bruch mit dem Zustand der Infantia auf der anderen Seite jedoch zu der Ansicht, dass der Übersetzung nicht die Möglichkeit gegeben ist, den anderen wiederzugeben, ohne auch im gleichen Zuge etwas auszusagen über diesen anderen (mit kritischem Charakter) sowie über sich selbst (mit reflexivem Charakter). Fazit: Die Übersetzung hat auch eine Stimme. Die Übersetzung sagt vieles aus. Und in der einmaligen Art und Weise, in der sie ihren Aussagen eine Stimme gibt, wird ihre Singularität konstituiert.

In Anbetracht dessen ist es unabdingbar zu berücksichtigen, dass der übersetzte Text nicht nur eine Lebensform des Originalwerks repräsentiert, sondern auch für sich allein und durch seine Singularität eine eigenständige Lebensform konstituiert $^{9}$. Und wenn wir dieser lebensnotwendigen Singularität der Übersetzung gegenüber nicht gleichgültig bleiben können, können wir auch nicht ignorieren, dass dies ihre Inschrift in der Ordnung der Zeit und in der Ebene der Geschichte impliziert.

\section{Antoine Berman und die Historizität der Übersetzung}

Im ersten Kapitel seines L'épreuve de l'étranger ${ }^{10}$, das sich der Erörterung der Gründungsrolle von Luthers biblischer Übersetzung in Deutschland widmet, verdeutlicht Antoine Berman ausgehend von der Lektüre eines Auszuges aus dem Aufsatz

9 Die Idee einer eigenen Form setzt hier nicht jedweden Modus der Essentialisierung dieser Eigenschaft des übersetzten Textes als diskursive Manifestation voraus. Im Kontext dieser Überlegung geht es schlichtweg darum, einen Übersetzungsbegriff als bloße Wiedergabeform einem Begriff der Übersetzung als einer Form zu gegenüberzustellen, die sich, um ihren Zweck der Repräsentation zu erfüllen, auch in Form einer Lebensform konstituieren muss, die hier immer als relationale Ordnung verstanden wird, das heißt: als eine Form, die ihre Singularität im Umfeld und im Ersuchen des Anderen begründet, als poietische Manifestation der und in der Beziehung zu einem Anderen.

10 Antoine Berman: L'épreuve de l'étranger. Culture et traduction dans l'Allemagne romantique. Paris: Gallimard 1984. 
Die Schrift und Luther von Franz Rosenzweig (1969), was er das Problem der allgemeinen Historizität der Übersetzung nennt. ${ }^{11}$ Berman geht davon aus, dass die Historizität eines Werkes (er bezieht sich auf die sogenannten Originalwerke), wenn auch nicht offensichtlich, zumindest nicht zu leugnen sei. ${ }^{12}$ Nun sei es jedoch zu überlegen, inwieweit man auch von der Historizität einer Übersetzung sprechen kann.

Zunächst erwägt Berman, dass man die Historizität eines universell übersetzten Werks nicht mit der Historizität seiner Übersetzungen verwechseln darf. Viele der kanonischen Werke, gros d'histoire - wie der Autor sagt - wurden nicht unbedingt mit einer Übersetzung betrachtet, von der man sagen könnte, sie sei historisch: „[...] celle qui fait époque en tant que traduction“"13. Hierfür, behauptet Berman, hätte eine dieser Übersetzungen in der Lage sein müssen, sich durch sich selbst hervorzuheben. Ich komme auf diesen Punkt zurück.

Ausgehend von einer epistemologischen Umschreibung, die an das benjaminische Denken anknüpft, schlägt Berman Folgendes vor: „[...] il convient d'appeler traduction historique celle qui fait époque en tant que traduction, celle où la traduction apparaît comme telle et accède ainsi, étrangement, au rang d'une œuvre, et non plus à celui d'humble médiation d'un texte lui-même historique. " ${ }^{14}$ Auf dieser Grundlage befürwortet der Autor eine zweite Unterscheidung: zwischen einer allgemeineren Historizität der Übersetzung - von der Übersetzung in ihrer instrumentalsten Bestimmung zur einfachen Vermittlung -, eines Zustands, in dem übersetzte Texte in der Geschichte eines bestimmten Werkes die Rolle einer bloßen Figuration spielen würden; und einer speziellen Historizität ${ }^{15}$, die man nur in seltenen Fällen anwenden würde, wo bestimmte Übersetzungen Protagonistenrollen einnehmen und sich selbst historisch machen würden.

Diese Unterscheidung stellt nun auch zwei verschiedene Arten dar, das Leben der Übersetzungen zu dimensionieren. Und an diesem Punkt muss Benjamins Unterscheidung zwischen einem Leben, das auf die Sphäre seiner eigenen Zwecke beschränkt ist, und einem Leben, das sich auf einer anderen Ebene, auf der Ebene der Geschichte, einschreiben kann, wieder aufgenommen werden: Bei den Übersetzungen, die die Grenzen eines Zustands einfacher Statisten ${ }^{16}$ nicht überschrei-

11 Franz Rosenzweig: Die Schrift und Luther. In: H.J. Störig: Das Problem des Übersetzens. Darmstadt: Wissenschaftliche Buchgesellschaft 1969, S.199-203.

12 Berman: L'épreuve de l'étranger, S. 51.

13 Ebd., S. 52.

14 Ebd.

15 Berman schlägt keinen Namen für diese zweite Form der Historizität vor, ich schlage ihn hier nur zum Zwecke der Übersichtlichkeit vor.

16 Auch diese Art des Verweises auf diese Übersetzungen wird hier, ausgehend von Bermans Vorschlag, nur zum Zwecke der Übersichtlichkeit genannt. 
ten - weil sie diese Möglichkeit der Transzendenz ignorieren, weil sie sich nicht erlauben, nicht wollen oder nicht in der Lage sind, dies zu tun - würde es sich um ein derart belangloses Leben handeln, dass es in der Tat kaum mehr als eine Lebensform bezeichnet werden könnte, höchstens durch das, was diese diskursive Manifestation von der Vitalität irgendeiner anderen Instanz repräsentiert - eine Dimensionierung ähnlich dem Zustand der lebenden Toten, auf die ich zuvor hingewiesen habe; bei den Übersetzungen hingegen, die den Status von Protagonisten erreichen, würde sich ihr Leben zumindest annähernd in den gleichen Begriffen dimensionieren wie das Leben anderer sogenannter Originalwerke.

Wenn also die erste Gruppe (die der Statisten-Übersetzungen) aus der absoluten Mehrheit der literarischen Übersetzungen besteht, sind die wenigen Beispiele, die Berman in der zweiten Gruppe (die der Protagonisten-Übersetzungen) erscheinen lässt - mit Sicherheit nicht zufällig - praktisch dieselben Beispiele, die bei Benjamin bereits als Ausnahmen erwähnt werden: Luthers Bibel, Voss’ Homer, Hölderlins Sophokles und so weiter.

Wie ich bereits erwähnt habe, ist das, was eine Übersetzung zu diesem besonderen Lebenszustand bringt, laut Berman, die Fähigkeit, sich hervorzuheben, auf die Außergewöhnlichkeit ihres Zustands als Übersetzung aufmerksam zu machen, die sie auf den Status eines Werkes erhebt. ${ }^{17}$ Jedoch war bei jedem dieser Ausnahmefälle, entweder aufgrund der Veröffentlichungen, die diesen Übersetzungen vorangingen (alle Neuübersetzungen), oder aufgrund der ästhetischen, kritischen, religiösen oder politischen Umstände, in die sich ihre Produktion sowie ihre Resonanz einschreibt, stets mehr von Bedeutung als nur die Besonderheit des Übersetzungszustands dieser Werke. Das heißt, nimmt man all ihre Eventualitäten und Umstände zusammen, gibt es keinen Zweifel daran, dass diese Übersetzungen in außergewöhnlicher Weise eine ganz besondere Form der Aufmerksamkeit bewirken konnten. Jedoch taten sie dies weder ausschließlich noch überwiegend durch die Offensichtlichkeit ihres Status als Übersetzung. In der Regel waren sie auf jenen Zustand der Infantia beschränkt, auf den alle Übersetzungen stets verwiesen wurden. Im Gegenteil: Wenn dies der Fall war, taten sie dies aufgrund dessen, was die Übersetzungen jenseits der Werke, die sie repräsentierten, zu bieten hatten. Sie taten dies nur durch das, was diese Übersetzungen über die Undurchsichtigkeit ihrer Beschaffenheit als Übersetzung hinaus darstellten, für das, was sie über jenes hinaus sagten, das sie als bloße Übersetzungen aussagen würden. Und wenn sie sich dadurch als bemerkenswert hervortaten, dann nur, weil sie - durch die Kraft der religiös-politischen Revolution (wie in Luthers Fall) oder durch die Wirkung der kritisch-ästhetischen Ungeheuerlich-

17 Berman: L'épreuve de l'étranger, S. 52. 
keit (wie in Hölderlins Fall), zu deren Vertretern sie wurden - einen Raum eroberten, in dem sie gehört wurden, in dem sie ihre Stimme geltend machten und sich auf diese Weise als lebenswürdige Formen erwiesen, die in der Lage sind, historisch zu sein.

In diesem Sinne ist es an dieser Stelle nicht notwendig, die Unterscheidung Bermans in Frage zu stellen, die an sich theoretisch sinnvoll und kritisch produktiv erscheint, sondern eher die Rechtfertigung, die Berman für seine Unterscheidung zwischen einer allgemeinen und einer speziellen Historizität der Übersetzungen findet, die der Autor, wie wir gesehen haben, in der Fähigkeit einer Übersetzung verortet, auf ihren außergewöhnlichen Status als Übersetzung aufmerksam zu machen. Und wir können dies tun, indem wir den Begriff des Lebens der Übersetzung in Bezug auf das, was aus der Lektüre von Benjamins berühmtem Aufsatz diskutiert wurde, neu skalieren.

Wenn wir also davon ausgehen, dass die Übersetzung neben dem, was sie für das ursprüngliche Werk darstellt, auch eine singuläre Lebensform darstellt und aus diesem Grund die Möglichkeit einer Infantia der Übersetzung nicht entsteht, müssen wir konsequenterweise annehmen, dass jede Übersetzung eine Stimme hat. Aus dieser Perspektive könnte die Rechtfertigung für die zuvor erwähnte Unterscheidung Bermans nicht einfach in der Anerkennung einer Stimme der Übersetzung liegen, da wir aufgrund ihrer vitalen Neudimensionierung davon ausgehen müssen, dass jede Übersetzung ihre eigene Stimme hat, was die Bedeutung der Stimme als unterscheidendes Merkmal unscharf werden ließe.

Um dieses Problem zusammenfassend zu formulieren: Meines Erachtens ist das Problem, dass es nicht ausreicht, eine Stimme zu haben, ebenso ist es notwendig, etwas zu sagen zu haben. Das heißt, da der Übersetzung nicht die Möglichkeit gegeben wird, keine Stimme zu haben, ist das, was tatsächlich zählt, die Art und Weise, wie jede Übersetzung ihre Stimme geltend macht. Und wenn wir diesen singulären Gebrauch der Stimme der Übersetzung mit dem kritischen Imperativ des faire-oeuvre ${ }^{18}$ von Berman in Verbindung bringen können, können wir schließlich sagen, dass Übersetzen immer auch eine Möglichkeit ist, Leben zu schaffen.

Um sich jedoch in diesem speziellen Zustand der (manchmal so außergewöhnlichen) Historizität zu unterscheiden, ist es immer noch notwendig, dass die Übersetzung neben der Anerkennung ihrer Stimme und dem, was sie zu sagen hat, einen minimalen Zustand des Gehörtwerdens erreicht. Es hängt also nicht nur von der kritischen Kraft der Übersetzung oder von ihrer Fähigkeit ab, sich bemerk-

18 Anton Berman: Pour une critique des traductions. John Donne. Paris: Gallimard 1995, S. 92: „,...] le traducteur doit toujours vouloir faire œuvre.“ 
bar zu machen, sondern auch - und in entscheidender Weise - von der Art, in der wir als Leser und Kritiker eine Beziehung mit dem übersetzten Text eingehen. Davon, wie sehr wir daran interessiert sind, den übersetzten Text als über das, was er vom Original wiedergibt - oder von einem bestimmten Verständnis dessen, was das Original ist - hinaus zu lesen. Es hängt davon ab, in welchem Maße wir bereit sind, es in seiner Singularität zu lesen, wie sehr wir in der Lage sind, es als eigene Lebensform und in seiner eigenen Zeit zu lesen, als eine bestimmte Inschrift in den Verlauf der Geschichte.

Erinnern wir uns daran, dass die von Benjamin und Berman erwähnten exemplarischen Übersetzungen diesen minimalen Zustand des Gehörtwerdens sicherlich erreicht haben. Es ist jedoch auch wichtig, sich daran zu erinnern, dass dieser besondere Zustand des Gehörtwerdens nicht ausschließlich durch den Erkenntnisgewinn geschaffen wird, den diese Übersetzungen als Übersetzungen darstellen, und dass sie auch nicht sofort von einem Tag auf den anderen entstehen. Hölderlins Übersetzungen, die von seinen Zeitgenossen als ungeheuerlich angesehen wurden - höchstwahrscheinlich aufgrund des Ausmaßes, in dem sie einen Bruch mit dem Standarderwartungshorizont angesichts einer literarischen Übersetzung im Allgemeinen repräsentierten, und mit der Übersetzung einer griechischen Tragödie (Sophokles) zu Beginn des 19. Jahrhunderts -, wurden erst zu Beginn des zwanzigsten Jahrhunderts, ausgehend von der besonderen Aufmerksamkeit, die ihr der Germanist Norbert von Hellingrath widmete, als innovativer Meilenstein der poetischen Übersetzung angesehen und in den folgenden Jahrzehnten wieder gelesen, aus der uns heute zeitgenössischen Perspektive. Auch die Übersetzung von Luthers Bibel, der so viele Autoren, wie Antoine Berman ${ }^{19}$, eine unverkennbare Gründerrolle der deutschen Sprache zuschreiben, der deutschsprachigen Literatur und der in Deutschland vorherrschenden Übersetzungstradition, wurde zu ihrer Zeit stark kritisiert. Es sei daran erinnert, dass Luther in einigen seiner Haupttexte zur Übersetzung ${ }^{20}$ Überlegungen anstellt, als Antwort auf die scharfe Kritik an seiner Arbeit als Übersetzer. Erst im Laufe des 16. Jahrhunderts, beeinflusst vom protestantischen Reformismus, wurde seine Übersetzung zu einem der Hauptinstrumente dieser theologisch-religiösen Revolution - mit einer eindeutig ideologischen, politischen und wirtschaftlichen Note -, wovon ausgehend in der ersten Hälfte des 17. Jahrhunderts einige der blutigsten Kapitel in der europäi-

19 Berman: L'épreuve de l'étranger, S. 43-60.

20 Unter diesen: Sendbrief vom Dolmetschen (1530) und Summarien über die Psalmen und Ursache des Dolmetschen (1531). Bezüglich Luther Übersetzungstheorie siehe unter anderem Mauri Furlan: A teoria de tradução de Lutero. In: Annete Endruschat/Axel Schönberger (Hg.): Übersetzung und Übersetzen aus dem und ins Portugiesische. Frankfurt am Main: Domus Editoria Europaea 2004, S. 11-21. 
schen Geschichte geschrieben wurden - wie die Reihe von Ereignissen, die heute als Dreißigjähriger Krieg bekannt sind - und im Laufe dessen sich die politischen Grenzen und Konturen eines ganzen Kontinents neu zeichneten.

Unter dem Gesichtspunkt eines übersetzerischen Denkens würde die Ungeheuerlichkeit - als Symptom - heutzutage darin bestehen, in den Szenen des Lesens und der Kritik des übersetzten Textes eine Denkweise zu verewigen, die rücksichtslos sowohl den Zustand der In-fantia der Übersetzung (in Form einer Nichtanerkennung ihrer eigenen Stimme) als auch des Zustands des Nichtgehörtwerdens (in Form der Gleichgültigkeit gegenüber dieser Stimme) nährt. Diese Zustände minimieren die lebenswichtige Größe einer Übersetzung, reduzieren sie auf die Negativität einer Form des Todes und verwandeln sie in eine Art lebenden Toten. Die Revolution hingegen - als Horizont -, sofern diese Bezeichnung zur Beschreibung der Veränderungen in den Denkweisen geeignet erscheint, würde in den Zeiten, in denen wir leben, eine Ethik des Gehörtwerdens durchlaufen, welche die Übersetzung in dem akzeptieren kann, was sie über die Erfüllung ihrer instrumentalen Bestimmung hinaus sagt: Sie würde eine Ethik des Gehörtwerdens durchlaufen, die die Übersetzung in dem, was sie als Lebensform zu sagen hat, annehmen kann.

\section{Exkurs: Von der Logik der Figuration zum Regime der Ko-Figuration}

Die bis hierhin skizzierten Unterscheidungen, die im Rahmen einer Neudimensionierung der Übersetzung getroffen wurden, stellen keine Art der Apologie jener dargestellten Lebensform dar, die sich aus der Übersetzung zum Nachteil dessen ergibt, was sie als Lebensform für das Original repräsentieren könnte. Die Anerkennung der Singularität der Übersetzung als Lebensform setzt also weder einen radikalen, relationalen Bruch mit dem ursprünglichen Werk noch eine Art Autonomisierung des übersetzten Textes ohne Berücksichtigung der Beziehungen, die für ihn konstitutiv sind, voraus - eine Voraussetzung, deren Begründung in den essentialistischen Diktaten nachhallt, die durch die verschiedensten Fronten zeitgenössischen Denkens radikal geschwächt wurden, insbesondere, sofern es sich bei dem, was in Frage gestellt wird, um die Autonomie eines Textes oder einer Reihe von Texten, die sich um den Status eines literarischen Werkes organisieren, handelt.

Anzuerkennen, dass der übersetzte Text jenseits des Repräsentierens einer Lebensform des Originals auch an sich und für sich selbst eine eigene Lebensform konstituiert, und in jenen Übersetzungen, die kritisch von ihrer Stimme Gebrauch 
machen, eine Bedeutung zu erkennen, die sie mit dem Status des Werkes errichtet, impliziert nicht zwangsläufig, dass diese Übersetzungen beim Lesen oder im kritischen Abhandeln als andere Werke behandelt werden sollten. Das Erkennen der Alterität des übersetzten Textes, einer Singularität, die ihn unterscheidet und den Unterschied ausmacht, bedeutet zunächst das Erkennen eines Anders-Werdens des Werkes, um hier eine deleuzianische Denkfigur zum Nachklingen zu bringen. Für die Kritik, die den übersetzten Text als Objekt nimmt, wird die sich daraus ergebende Perspektive immer mindestens eine zweifache sein: das Lesen des übersetzten Textes als Repräsentation des Originalwerks, in den uns bekannten benjaminischen Begriffen; und das Lesen des übersetzten Textes als eine einmalige, singuläre Lebensform, die von der ursprünglichen Arbeit ausgeht und die ursprüngliche Arbeit weitergibt, durch die jedoch auch etwas Neues entspringt.

Die benjaminsche Logik der Übersetzung als Repräsentation oder Figuration des Lebens des literarischen Werkes, welche sich stark auf das immer noch vorherrschende Verständnis sowohl der Beziehungen der Übersetzung zur Zeit als auch ihrer vitalen Dimensionierung auswirkt, stößt angesichts der Annahme, dass sowohl der ursprüngliche als auch der übersetzte Text eigenständige Lebensformen sind, an ihre Grenzen. Wenn von diesem Standpunkt aus eine Übersetzung nicht mehr nur die Figuration der Singularität eines anderen Textes ist, sondern eine Beziehung zwischen zwei Singularitäten, ist es notwendig, von diesem neuen Verständnis ausgehend auch das etablierte Beziehungssystem zu überdenken.

In diesem Zusammenhang bietet es sich an, an den sogenannten Schematismus der Ko-Figuration zu erinnern, eine von dem Komparatisten Naoki Sakai im Zentrum seiner Reflexion über die Beziehungen zwischen sprachlichen, nationalen und kulturellen Einheiten entwickelte Denkfigur, die an das angelehnt ist, was er als bordering turn bezeichnet:

The unity of a language is represented always in relation to another unity; it is never given in and of itself but in relation to an other. One can hardly evade dialogic duality when determining the unity of a language; language as a unity almost always conjures up the copresence of another language, precisely because translation is not only a border crossing but also and preliminarily an act of drawing a border, of bordering. Hence I have to introduce the schematism of configuration in analyzing how translation is represented. ${ }^{21}$ (meine Hervorhebung)

21 Naoki Sakai: Translation and the Figure of Border: Toward the Apprehension of Translation as a Social Action, in: Profession (MLA), New York, 2010, S. 25-34, hier S. 32. Verfügbar unter: www. jstor.org/stable/41419858. Letzter Zugriff am 10.10.2017. 
Mit anderen Worten, nach Sakais Ansicht wäre es überhaupt nicht möglich, die Übersetzung ohne Erwägung ihrer Gründungsfunktion mit dem Original zu denken. Es sei an Folgendes erinnert: Übersetzung (im Sinne des übersetzten Textes) nennen wir einen bestimmten Text, wenn wir diese Beziehung als ihren Gründer betrachten (oder voraussetzen), die ihn aus einem anderen Text heraus gründet; Original (im Sinne des Originalwerks) nennen wir einen bestimmten Text, wenn wir jene grundlegende Beziehung betrachten (oder voraussetzen), die einen anderen Text begründet. Das heißt, ohne die Bedeutsamkeit dieser Übersetzungsbeziehung zu vernachlässigen, ist es notwendig, daran zu erinnern, dass außerhalb dieser Beziehung, jenseits dieser Beziehung zwischen Originalwerk und übersetztem Text zwei einzelne Texte bestehen, die sich als solche auf ihre Weise in ein weites, relationales Universum einfügen wie jeder beliebige andere Text.

Daher kann von Ko-Figuration nur im Rahmen eines Relationssystems gesprochen werden, das es zulässt, diese beiden als Übersetzung und Original bezeichneten Texte als zwei Formen der Singularität in Beziehung zueinander zu setzen.

In diesem Sinne stellt sich das Regime der Ko-Figuration als eine Möglichkeit heraus, die benjaminsche Logik neu zu formulieren, wonach die Übersetzung, die sich darauf beschränkt, eine fremde Lebensform zu repräsentieren, auf das Paradigma der Figuration des Originals beschränkt wäre. Im Regime der Ko-Figuration wird angenommen, dass sowohl das Original als auch die Übersetzung singuläre Lebensformen bilden, ohne dass dasjenige, was beide Texte für den jeweils anderen repräsentieren, verloren geht.

\section{Veredas der Übersetzungsbeziehungen: im Tanz der Typoskripte}

Um hier auf die berühmte Formel von Koselleck zurückzukommen könnte man sagen, dass die Schrift - sowohl das Originalwerk als auch der übersetzte Text bereits ab dem Moment der Einschreibung in den Beziehungsraum, der sie konstituiert, den Zustand einer Form der „vergangenen Zukunft“ einnimmt. ${ }^{22}$ Während es ihr nicht möglich ist, zeitweilig mit sich selbst übereinzustimmen, oder vielmehr, während es ihr nicht möglich ist, eine Zeitform zu sein, die mit jener Zeitform übereinstimmt, welche sie selbst im Moment ihrer Lektüre gründen kann, ist

22 Vgl. Reinhart Koselleck: Futuro passado: contribuição à semântica dos tempos históricos, übersetzt von Wilma Patrícia Maas, Carlos Almeida Pereira. Rio de Janeiro: Contraponto, Ed. PUC-Rio 2006. 
die Schrift per definitionem außerzeitlich sich selbst gegenüber und konstituiert sich deshalb als eine Zeit des Lebens, die immer auch in gewissem Maße eine Zeit des Todes ist.

Es ist wichtig anzumerken, dass dies Themen sind, die wir Leser im Moment der Lektüre sowohl des übersetzten Textes als auch des Originalwerks ernsthafter angehen müssen, wenn wir den erkenntnistheoretischen Rahmen berücksichtigen, der hier gezeichnet wird. Wir werden dann in dem, was wir normalerweise Übersetzung nennen - was in seiner sprichwörtlichen Negativität normalerweise als Todesform stigmatisiert wird - auch dessen Lebensformen anerkennen müssen; so wie wir in jenem, was wir als das Originalwerk bezeichnen - dass sich traditionellerweise als beständige Lebensform verewigt, auch ihre Todeszeiten anerkennen müssen.

Oder anders ausgedrückt, dies ist eine Möglichkeit, die Herausforderung zu formulieren, die sich heute denjenigen auferlegt, die sich in ein so reichhaltiges, weitläufiges und komplexes Relationsgefüge begeben wie das der Übersetzungen von João Guimarães Rosas Werk in andere Sprachen. Dies liegt daran, dass unter bestimmten Sichtweisen des kritischen Lesens von Übersetzungen, ganz anders als hier vorgeschlagen, dasjenige, was normalerweise als echter Erkenntnisgewinn hervorgebracht wird, bestenfalls eine detaillierte Bewertung dessen ist, wie viel jede Übersetzung vom Originalwerk für Leser anderer Sprachen bieten kann. Und da die Übersetzungsökonomie aus dieser Perspektive immer negativ auf die endgültige Bilanz der Bewertung des übersetzten Textes einwirkt, führt diese Art kritischer Auseinandersetzung normalerweise zu Narrativen, die sich im großen Inventar der Fehlschläge eines jeden Übersetzungsunterfangens gründen, während die übersetzten Texte auf eine prekäre und reduzierende Form der Repräsentation des Werkes naturalisiert werden, welches ihre Entstehung hervorrief.

Vielleicht können wir akzeptieren, dass solche Perspektiven, wie sie oben beschrieben wurden, ihren Platz im Umgang mit der Übersetzung von Texten haben, die eher instrumenteller Natur sind - wie zum Beispiel Anleitungen oder Texte mit vorwiegend informativem Umfang. In diesen Fällen entwickelt sich Kritik im Hinblick auf das, was wir angemessener Weise als Evaluierung bezeichnen. Diskussionen, die sich auf die Bedeutung von Unterschieden konzentrieren wollen - wie etwa diejenigen, die uns im Bereich der literarischen Übersetzung interessieren - machen dann Platz für Diskussionen zur Minimierung der Unterschiede, zur Optimierung der Kommunikation und zur Aufrechterhaltung eines Qualitätsstandards.

Obwohl wir sagen können, dass jede Übersetzung eines literarischen Werkes auch den instrumentellen Zweck erfüllt, das Werk in eine andere Sprache zu übersetzen, wird die literarische Übersetzung nicht auf diese Dimension der Instru- 
mentalität reduziert, schlichtweg weil Literatur - der Gegenstand dieser Übersetzungspraxis - sich auch nicht auf diese instrumentelle Dimension reduziert. Wenn wir also sagen können, dass Curt Meyer-Clasons Übersetzungen von Guimarães Rosas Werk den wichtigen Zweck erfüllen, dieses brasilianische Literaturwerk einem deutschen Lesepublikum anzubieten, treibt uns die Berücksichtigung des hier vorgeschlagenen erkenntnistheoretischen Rahmens auch dazu an, in Erwägung zu ziehen, dass diese Übersetzungen nur deshalb funktionieren, weil sie eine einmalige Form dieser brasilianischen Werke auf Deutsch darstellen, ein Anders-Werden von Rosas Werken auf Deutsch - mit anderen Worten: eine andere Lebensform, die sich hier als spezifisches Objekt dieser kritischen Perspektive umschreibt.

Wenn wir aus dieser Perspektive eine Arbeit aufmerksamer Lektüre über die Art und Weise erwarten können, wie Rosas Texte mit den veröffentlichten Texten wie etwa seiner Übersetzungen zusammen leben, und dabei die Beziehungdynamik zu identifizieren versuchen, die dieses System des Zusammenlebens gründet - im Übrigen mit der gleichen relationalen Komplexität, die wir unter den Werken und Übersetzungen zahlreicher anderer Autoren und Übersetzer entdecken können -, öffnet der Reichtum eines Archivs wie das von Curt Meyer-Clasons Nachlass, der derzeit im Iberoamerikanischen Institut in Berlin hinterlegt ist, noch eine Reihe weiterer Arbeitsperspektiven.

Eine davon betrifft die Einordnung, die Transkription und die Diskussion der Briefwerke dieses Autors oder seines Übersetzers, wie etwa die Arbeit, die der Öffentlichkeit im Jahre 1997 in Form einer Masterarbeit und 2003 in Buchform die fruchtbare Korrespondenz zwischen Meyer-Clason und Rosa über fast ein Jahrzehnt zur Verfügung stellt, von 1958 bis $1967 .{ }^{23}$ Der Reichtum dieses Materials ist insbesondere aus der Sicht der Feststellungen des Autors über sein eigenes Werk berüchtigt; sein Wert wird jedoch nicht nur darüber definiert, wie viel dieses Material in Form von Aufschlüssen für eine Vertiefung des Lesens und Studiums von Rosas Arbeit mitbringen kann. Die Korrespondenz zwischen Autor und Übersetzer ermöglicht es uns auch, Narrative dieser Beziehung zu rekonstruieren, indem wir andere Aspekte dieser Geschichte des Zusammenlebens erblicken können, und zwar aus einer Perspektive, die die Textbeziehungen zwischen übersetztem Text und Originalarbeit überschreitet - obwohl es sich fast immer um diese dreht.

Es gibt jedoch auch eine andere Arbeitsperspektive, die sich aus einem noch selteneren und kostbareren Material ergibt, das das Briefmaterial einschließt und

23 João Guimarães Rosa: João Guimaraes Rosa: correspondência com seu tradutor alemão Curt Meyer-Clason (1958-1967), Herausgabe, Organisation und Anmerkungen con Maria Aparecida Faria Marcondes Bussolotti, Übersetzung Erlon José Paschoal. Rio de Janeiro: Nova Fronteira; Belo Horizonte: Ed. da UFMG 2003. 
das bis heute erst sehr zaghaft untersucht wurde: Ich beziehe mich auf die Typoskripte der Übersetzungen von Meyer-Clason, von Rosa überschrieben und an den Seitenrändern kommentiert. Meyer-Clason pflegte, die ersten Fassungen seiner Übersetzungen abzutippen, die er per Post an Rosa schickte. Nachdem er jede Version gelesen und kommentiert hatte, schickte er seinem Übersetzer die gleichen kommentierten Anmerkungen (im Allgemeinen mit Bleistift) zurück, normalerweise zusammen mit einem Brief. Denn es ist diese herumgekommene Sammlung von Typoskripten, die dieses Material formen, welche es in der Bibliothek des Iberoamerikanischen Instituts in Berlin noch zu entdecken gilt.

Wenn zum einen die Korrespondenz es uns erlaubt, Aspekte der Beziehung zwischen diesen Autoren und Übersetzern zu rekonstruieren, fast immer auf der Grundlage eines Aspekts einer laufenden Übersetzungsbeziehung, und wenn wir andererseits davon ausgehen können, dass jede veröffentlichte Übersetzung eine Art Ergebnisprotokoll dieser Übersetzungsbeziehungen darstellt, stellt diese Sammlung überschriebener Typoskripte eine Art Körper-an-Körper dieser Übersetzungsbeziehung in ihrem Geburtsprozess dar. Einige dieser Notizen und Bemerkungen von Rosa sind im eigentlichen Hauptteil seiner Briefe zusammengefasst, aber die Eingriffe in einige der diskutierten Texte gehen weiter. Es ist, als könnten wir in einer Zeit, die die Zeit der Übersetzungsbeziehung jedes Textes ist, einen Tanz beobachten, in dem der Autor auf jeden Schritt seines Übersetzers reagiert, der den Autor mal begleitet, indem er sich führen lässt, mal ihn zu führen versucht.

Leser und Forscher, die diese Veredas der Übersetzungsbeziehung erforschen wollen, müssen die Typoskripte in ihren verschiedenen Zeiten aufblühen lassen um abschließend die Kraft jener speziellen Blume des Verses von Paul Celan in dem Epigraph hier wieder aufzunehmen. Nicht im Sinne einer Rückführung jedweder Bewegung der Übersetzung auf das Originalwerk - schließlich ist es keine Wiederherstellung des Ursprungs, die in diesem Übersetzungsvorgang behandelt werden muss. Vielmehr muss man lernen, in jedem Schritt dieser typoskriptischen Choreographie die Todeszeiten und die Lebenszeiten einer kommenden Übersetzung zu lesen: die Singularität einer deutschen Sertão-Welt, die in diesen Schriften ihre Veredas noch in statu nascendi erprobt.

Es geht hier also weniger um eine methodologische als um eine erkenntnistheoretische Frage. Zuallererst müssen wir lernen, die Übersetzung mit anderen Augen zu sehen, mit anderen Ohren zu hören. Und dies wird nur möglich sein, wenn wir uns bereit zeigen, auch die Übersetzung als eine singuläre Blume anzunehmen, die durch die Überwindung ihrer naturalisierten Infantia, zu der sie für gewöhnlich verurteilt wird, nicht in einer vorgegebenen Zeit, nicht in einer vergangenen Zeit und auch nicht in einer verheißenen Zeit erblüht, sondern in Zeiten, die sich immer als ihre eigenen erweisen. 